\title{
A Client Puzzle Based Public-key Authentication Protocol
}

\author{
Chun-Kan Fung, M.C. Lee, and M.Y. Wong \\ Department of Computer Science and Engineering \\ The Chinese University of Hong Kong, Shatin, N.T., Hong Kong \\ \{ckfung,mclee,mywong\}@cse.cuhk.edu.hk
}

\begin{abstract}
Network denial-of-service attacks, which exhaust the server resources, have become a serious security threat to the Internet. Public Key Infrastructure (PKI) has long been introduced in various authentication protocols to verify the identities of the communicating parties. Although the use of PKI can present difficulty to the denial-of-service attackers, the underlying problem has not been resolved completely, because the use of public-key infrastructure involves computationally expensive operations such as modular exponentiation. An improper deployment of the public-key operations in a protocol allows the attacker to exhaust the server's resources. This paper presents a public-key based authentication protocol integrated with a sophisticated client puzzle, which together provides a good solution for network denial-of-service attacks, and various other common attacks. The basic strategy to protect against denial of service is to impose an adjustable cost on the attacker while it launches the attacks. The proposed client puzzle protocol can also be integrated with other network protocols to protect against denial-of-service attacks.
\end{abstract}

Keywords: Authentication Protocols, Client Puzzles, Denial of Service, Network Security, Attacks.

\section{INTRODUCTION}

Denial-of-Service (DoS) attack is characterized by an explicit attempt of the attackers to prevent legitimate users of a service from using that service [1]. There has been a growing concern about this attack in recent years, 
especially after the series of denial-of-service attacks on the Internet on February 2000 [2].

Denial-of-Service attacks can be classified into three types. These include (i) consumption of scarce resources; (ii) destruction or alteration of configuration information; and (iii) physical destruction or alteration of network components [1]. Any protocol where the server commits extensive computations or memory allocation prior to or as a part of client authentication is vulnerable to network DoS attacks [3], which corresponds to the first type of denial-of-service attack above. The basic strategy in handling the denial-of-service attack is that the server should require the client to commit its resources before extensive resources could be committed to the client in an authentication protocol session.

TCP connection protocol was reported to be vulnerable to SYN flooding attack in 1996 [4]. This is one of the well-known DoS attacks against a network protocol. During the attack, the attacker sends a huge number of SYN messages to the server to initialize connections and then leaves the subsequently established connections unattended. Since the server allocates buffer space right after the reception of a SYN message, memory of the server can thus easily be exhausted by such connection requests. Juels and Brainard [5] pointed out that the SSL protocol [6] is vulnerable to a similar form of attack. However, it is the CPU resource rather than the memory space, which could be exhausted rapidly because expensive cryptographic operations are required on the server-side in the SSL protocol.

Entity authentication has long been used in communication protocols to tackle different attacks, e.g. the man-in-the-middle attack. Authentication based on the public-key infrastructure is computationally expensive, as the underlying cryptographic operations such as the modular exponentiation involve extensive computations. An attacker may be able to exhaust the computational or memory resources of its target if the underlying authentication or communication protocol is improperly designed. For instance, $\mathrm{Ng}$ and Tan's protocol [7] is one that is vulnerable to the resource consumption attack; the server is required to perform public-key decryption right after the reception of the client request message in this protocol. There are several other protocols identified as being vulnerable to this type of attack [8] [9] [10].

Dwork and Naor first introduced the concept of client puzzle to combat junk mail attack [11]. A client puzzle is a small cryptographic problem created by the server in response to a client request. The client should first commit its resources to solving the puzzle before completing the remaining part of the communication protocol. While the legitimate users could experience only a slight degradation of service under this scheme, a big cost will be imposed on a denial-of-service attacker who tends to create a huge 
number of requests within a short duration, because the attacker is required to solve a unique puzzle for each of its service requests. With appropriate adjustment on the puzzle difficulty level, the client puzzle method can handle relatively fast attacks and allow for graceful service degradation. Aura, Nikander and Leiwo [12] proposed a client puzzle and applied it to an authentication protocol. The client puzzle used has an inherent problem that the solution to a puzzle may not exist since the protocol does not verify the existence of a solution during puzzle construction. A later authentication protocol designed also by Aura, Nikander and Leiwo [3] employs a puzzle without the above problem. However, similar to the approach proposed by Matsuura and Imai [10], the puzzle does not have a difficulty level; so graceful degradation of service would not be possible. The authentication protocol is also vulnerable to denial-of-service attack because the puzzle is not created in a stateless way. Juels and Brainard from the RSA Laboratories proposed a client puzzle protocol to address the TCP SYN attack [5]. This client puzzle protocol does not have the above-mentioned problems; however, there are still other identified defects.

In this paper, we propose an enhanced version of the client puzzle protocol of Juels and Brainard [5]. The defects in Juels and Brainard's protocol will be identified and removed from our client puzzle solution. In addition, a public-key based authentication protocol integrated with our client puzzle protocol would be proposed. The paper further demonstrates how the proposed integrated authentication protocol can resist effectively the network DoS attack and other types of common attacks.

\section{AN IMPROVED CLIENT PUZZLE PROTOCOL}

This section presents our client puzzle protocol for handling the denialof-service attacks. This client puzzle can be added on top of an existing network protocol in order to protect the protocol against network DoS attacks.

\subsection{Protocol Descriptions}

Consider the use of our client puzzle to protect a protocol $M$ against the network denial-of-service attacks. To initiate the execution of the protocol $M$, the client submits its unique identity $C$ in the form of a request message to the server.

The server then checks the availability of system resources in order to determine the difficulty level $k$ of the client puzzle to be sent out. The resource availability may refer to the availability of memory or CPU 
resource or both. Normally, the difficulty level is inversely proportional to the availability of the system resources, allowing a graceful degradation of services during the denial-of-service attacks. In a normal situation, $k$ is set to zero, and no puzzle needs to be solved by the clients.

After the determination of the difficulty level, the puzzle would be constructed using two one-way, collision-resistant hash operations. First, a bit-string $\mathrm{zl}$ is generated by the hash operation $h(C, s, t)$, where the hash function $h$ would operate on a single input bit-string formed by the concatenation of the parameters $C, s$ and $t$. Then a second hash operation is performed on $\mathrm{zl}$ to produce another bit-string z2. The client identity $C$ is included in the hash input to make the puzzle client specific so that a solution, for a given puzzle, found by one client cannot be used by other clients. Parameter $s$ is the server secret key, which should be long enough (e.g. 128 bits) to prevent brute force attacks. In order to impose a limit time for solving the puzzle by the client, the puzzle should be associated with a timestamp, $t$, which is set to the current date and time with a precision up to a second. The client needs to solve the puzzle before it expires; otherwise, the protocol execution will be terminated no matter whether the returned solution is correct or not.

Assume the hash output is of length $L$ bits. The puzzle is made up of the hash image $\mathrm{z} 2$, the partial pre-image $\mathrm{z} 1<k+1, L>$, i.e. the $(k+1)^{\text {th }}$ to the $L^{\text {th }}$ bits of $z 1$, the timestamp $t$ and the difficulty level $k$. In this way, the puzzle can be constructed in a totally stateless fashion. It is important to commit as little resource as possible in the construction of a puzzle because the DoS attackers intend to exhaust the server resources.

To solve the puzzle, the client finds by brute force a $k$-bit string $z 1^{\prime}<1, k>$, which, when concatenated with the given partial pre-image $z 1<k+1, L>$, satisfies the following relation:

$$
\mathrm{z} 2=h\left(\mathrm{z} 1^{\prime}<1, k>, \mathrm{z} 1<k+1, L>\right)
$$

After solving the puzzle, the solution $z 1^{\prime}<1, k>$ together with the received $\mathrm{z} 1<k+1, L>, t$ and $k$ and the client identity $C$ would be sent back to the server for verification.

Before verifying the puzzle solution, the server first checks the timestamp $t$ to see if the puzzle has expired. If $T$ is the maximum allowable time for the client to solve the puzzle and to return the solution, the server must ensure that $\tau-t \leq T$ where $\tau$ is the current date and time on server side. If the timestamp has not expired, the server performs the same hash operation during puzzle construction using the client-submitted $C$ and $t$ to get the bitstring $m=h(C, s, t)$. The last $L-k$ bits of this bit-string (i.e. $m<k+1, L>$ ) are compared with the received $\mathrm{z} 1<k+1, L>$ to make sure that the timestamp and 
the client identity are the same as the original ones. After that, the clientpurported solution is verified by checking the equality between $h(m)$ and the output from the hash operation on the bit-string formed by the concatenation of $z 1^{\prime}<1, k>$ and $m<k+1, L>$. If the equality holds, the solution can be assured to be correct; the server can then commit its resources to the client, otherwise, the request is rejected.

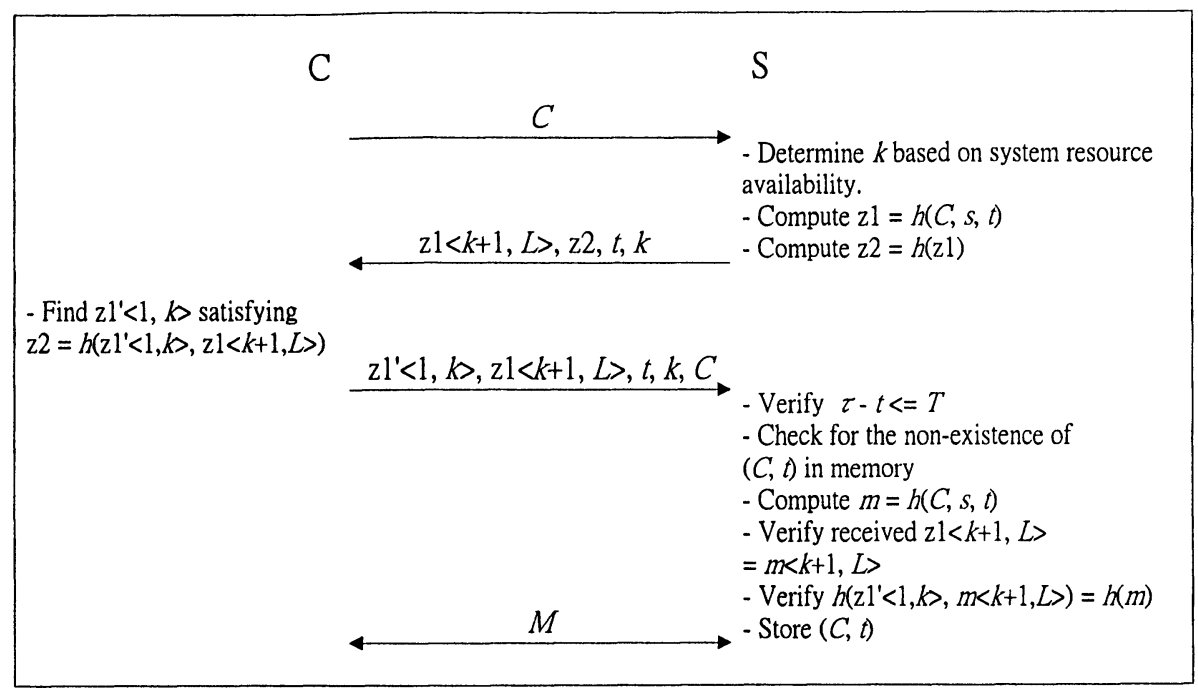

Figure 1. The client puzzle protocol

Prevention of the pre-computation of puzzle solution is a basic puzzle design issue. In our client puzzle protocol, a timestamp $t$ and a long server secret key $s$ are the parameters used in creating the client puzzle. This makes the puzzle component $\mathrm{z} 1<k+1, L>$ unpredictable to the client; and so, the solution cannot be pre-computed. However, if the time parameter $T$ is set to be too large, a client (or attacker) can submit the same solution a number of times before the puzzle expires. So the server should register the pair $(C, t)$ in memory after the solution has been verified correct. This indicates that client $C$ has submitted a correctly solved puzzle created at time $t$. Before verifying a given solution from any client $C$, the server should check to ensure that there is not any pair of the form $(C, t)$ in memory for the client as depicted in Figure 1. This pair $(C, t)$ does not need to be kept in memory for a long time; it will be removed once the puzzle has expired. In theory, upon confirming a given puzzle solution, if $\tau-t=T$, then no pair of the form $(C, t)$ needs to be kept for client $C$. However, if $\tau-t<T$, the duration for which $(C$, $t)$ needs to be stored is $(T-(\tau-t))$, which is usually a short period. 


\subsection{Comparisons and Improvements}

In the client puzzle protocol proposed by Juels and Brainard [5] (referred to as the Juels-Brainard protocol hereafter), a puzzle can be made up of $n$ ( $\geq$ 1) sub-puzzle(s) each having the same difficulty level $k$. The probability for an attacker to guessing the solutions correctly is $1 / 2^{k n}$. To reduce this probability to a certain level, the value of $n$ can be increased. However, the same level can be achieved by increasing the value of $k$ with a smaller $n$. The latter approach has the advantage of having a smaller overhead in puzzle transmission because the puzzle size is smaller with a smaller $n$. Another advantage is the lower cost in puzzle creation and verification because the number of sub-puzzle creation and verification is smaller with a smaller $n$. It is important to commit as little resource as possible in puzzle creation and verification by keeping $n$ small in order to prevent DoS attack. The cost of this approach is that the average work factor $\left(1+2^{k}\right) n / 2$ to a client in solving the puzzle is higher for the same $1 / 2^{k n}$. Our puzzle can be viewed as made up of one single sub-puzzle $(n=1)$. However, it can be easily extended to one consisting of more than one sub-puzzle by adding an additional sub-puzzle index to the input parameter list of the hash operation during the puzzle construction stage.

To allow for a fair comparison, a puzzle of the Juels-Brainard protocol is meant hereafter to be one consisting of one sub-puzzle only, i.e. $n=1$.

In the Juels-Brainard protocol, the authors claim that a collision-resistant hash function is not a requirement. However, their puzzle solution verification process does not take this claim into account. In the puzzle creation stage of the Juels-Brainard protocol, a bit-string $x$ is computed by the server as a hash of a set of service parameters and a server secret key. Then another bit-string $y$ is computed as a hash of $x$, i.e. $y=h(x)$. The server then sends this $y$ and part of $x$ to the client who is required to find the remaining part of $x$ by brute force using the equation $y=h\left(x^{\prime}\right)$. In the verification stage, the server generates $x$ again and compares it against the client-purported solution $x^{\prime}$ to determine the correctness of this solution. However, a non-collision-resistant hash function means that apart from the server-generated $x$, there could be another $x^{\prime}$ satisfying the equation $y=h\left(x^{\prime}\right)$. So the Juels-Brainard protocol could treat a correct solution as wrong. In our client puzzle protocol, the client-purported solution will not be compared directly with the server-generated $x$. Instead, an additional hash operation would be performed on the client-purported solution to see whether the output is equal to $y$. With this modification, a correct solution will not be marked as incorrect even if a non-collision-resistant hash function is used. This modification would also be useful when using a collision-resistant hash function because collision-resistant is not a guaranteed property. Collision 
could still occur in some claimed collision-resistant hash functions, e.g. MD4 [13].

In the Juels-Brainard protocol, the client needs to submit only the found leading $k$-bit sub-string of $x$ as solution; the remaining $L-k$ bits of $x$ are to be computed by the server during solution verification. When compared with our approach, their solution would be $L-k$ bits shorter. However, it is inappropriate to rely on the server to calculate the remaining $L-k$ bits. Consider the case with the puzzle difficulty level $k$ equal to 1 . After solving the puzzle, instead of submitting the solution immediately, the client stores the solution for later use. Suppose after the puzzle has expired, the client retrieves the solution from storage and submits it. The client knows that the server could possibly reject the solution as the puzzle has expired. So, before submission, it updates the timestamp $t$ in order to cheat the server. On the server side, the 1-bit solution is computed using this fake timestamp, and is then compared with the client-purported solution. Although the timestamp has been modified, the probability of producing the same 1-bit solution as the client-purported one is still very high. The problem is that the solution is very short. In this example, this probability is $1 / 2$. For $k=2$, this probability is $1 / 2^{2}$. In general, this probability is $1 / 2^{k}$. We can see that the lower the value of $k$, the more severe would be the problem. So one possible solution is to set a bigger lower bound for $k$ (or $n$ in the Juels-Brainard protocol). However, this makes graceful degradation of service difficult and the clients will experience a large degradation of service quality in a short time at the beginning of a potential DoS attack. Therefore, we require the client to submit both the solution and the received bit-string $\mathrm{z} 1<k+1, L>$ instead of the solution $\mathrm{zl} 1^{\prime}<1, k>$ alone in our protocol. The server should check whether the received bit-string $\mathrm{z} 1<k+1, L>$ matches the server-computed bitstring $m<k+1, L>$ before verifying the solution. With such modifications included in our protocol, we consider again the probability of producing a matched $m<k+1, L>$ given the submitted $\mathrm{z} 1<k+1, L>$ when the timestamp has been modified. Among the $2^{L}$ possible values of $m, 2^{k}$ of them have the sub-string $m<k+1, L>$ matching the given $z 1<k+1, L>$. Assume the hash outputs are uniformly distributed. The probability of producing a bit-string $m$, using the fake timestamp, with the last $L-k$ bits (i.e. $m<k+1, L>$ ) matching the client-submitted $\mathrm{z} 1<k+1, L>$ is $1 / 2^{L-k}$. According to this probability $1 / 2^{L-k}$, the higher the value of $k$, the higher would be the chance of the matching when the modified timestamp is being used to generate the bit-string $m$. To make this probability close to zero, we suggest the value of $k$ to be between 0 and $L / 2$; and $L$ should have a length of at least 128 bits. In fact, the current common hash functions such as MD5 and SHA have a hash output with a length not less than 128 bits. 
Before a puzzle expires, a client could submit the same solution a number of times, leading to multiple allocations of server resources. In the JuelsBrainard protocol, it has a mechanism to prevent multiple allocations of buffer resources. This is done by associating a unique identifier derived from the first request message ( $M_{i}^{l}$ in Juels-Brainard protocol notation) to the corresponding allocated buffer slot; and the server ensures that only one buffer slot would be allocated for each request. However, the authors assume the unavailability of PKI in the design of their protocol because they claimed that PKI could effectively prevent denial-of-service attacks. And no additional benefits could be achieved when using client puzzle in the presence of PKI. In fact, this is not true because the computationally expensive operations such as modular exponentiation in PKI could open up the CPU exhaustion attack. The client puzzle protocol could be used to protect a public-key based authentication protocol. In this case, after verifying the puzzle solution, the server may need to perform the expensive public-key cryptographic operations in executing the subsequent authentication protocol. In our protocol, instead of relying entirely on the client request message for the unique identifier, we make use of the high precision requirement (at least up to a second) of timestamp. The unique identifier is derived from the client unique identity $C$, which appears in the first request message, and the puzzle timestamp $t$, which is generated by the server, to form the unique pair $(C, t)$. This mechanism can reduce the number of parameters in the client request message to a minimal. Whenever a solution has been verified correct, the unique pair $(C, t)$ is stored in memory until the corresponding puzzle expires. As depicted in Figure 1, the server needs to check for the non-existence of the unique identifier $(C, t)$ for each service request before verifying the puzzle solution. To allow for a rapid search for the existence of an identifier, bucket hashing on the identifiers could be employed. If the existence of a submitted identifier $(C, t)$ can be found, the protocol should be terminated immediately and both CPU and memory resources are not allocated. If the client submits a fake $(C, t)$, it cannot pass the subsequent solution verification. Therefore, if a client (or attacker) reuses the same solution before the puzzle expires, the solution will be rejected. Thus multiple allocations of both extensive CPU and memory resources would not occur.

\section{THE PROPOSED AUTHENTICATION PROTOCOL}

The proposed client puzzle protocol can be integrated with or added on top of a network protocol which it protects. In this section, we propose a 
public-key authentication protocol, and demonstrate how our client puzzle protocol can be securely and properly integrated with the protocol which it protects. If the integration of the client puzzle and the network protocol is not done properly, not only the denial-of-service attacks cannot be prevented, and the underlying protocol may be liable to further security attacks.

\subsection{Notations}

Let $k_{\Sigma}$ be the public key of the entity $\sum$, and $k_{\Sigma}{ }^{-1}$ be the corresponding private key. $E\{w\} k_{\Sigma}$ denotes the encryption of message $w$ using the key $k_{\Sigma}$. $N_{\Sigma}$ is a random nonce generated by entity $\Sigma$. The other notations are the same as those in the client puzzle protocol.

\subsection{Protocol Descriptions}

The proposed mutual authentication protocol is a challenge-response and timestamp-based authentication protocol. Though a timestamp parameter is employed in the protocol, clock synchronization is not required. The proposed authentication protocol consists of 4 message exchanges between the client and the server. The first two are basically the same as the first two messages in the client puzzle protocol depicted in Figure 1.

It is assumed that the communicating entities have authentic copies of all the required public keys. The authentication protocol begins by client $C$ sending an authentication request message to the server $S$.

\section{Message 1: $C \rightarrow S: C$}

Message 1 of the authentication protocol, the same as the request message in the proposed client puzzle protocol, is used as the authentication request message. In this message, client identity $C$ is used as the only parameter to formulate the authentication request. It serves as a hello message only; and does not trigger the execution of expensive computations or the allocation of memory resources by the server. Otherwise, the integrated protocol could not protect against DoS attacks.

After the reception of the client request, the server performs the same tasks as outlined in the client puzzle section. The tasks include the determination of the puzzle difficulty level $k$ based on the availability of system resources, and the construction of the client puzzle. If $k$ is set 0 , the computation of $\mathrm{zl}$ would still be necessary. As mentioned in the client puzzle protocol, the string $\mathrm{z} 1<k+1, L>$ can be used to validate the timestamp in message 3 on the server side. So it is important to ensure message freshness for protection against replay attacks. 


\section{Message 2: $S \rightarrow C: \mathrm{z} 1<k+1, L>, \mathrm{z2}, t, k$}

The second message is also the same as the second one in the client puzzle protocol. The server passes the formulated strings $\mathrm{z} 1<k+1, L>$ and $\mathrm{z} 2$ (for $k \neq 0$ ) together with the timestamp $t$ and difficulty level $k$ to the client.

On reception of message 2 , if the difficulty level is greater than zero, the client finds a solution to the puzzle by brute force. Otherwise, no puzzle needs to be solved.

\section{Message 3: $C \rightarrow S: \mathrm{z1}^{\prime}<1, k>, \mathrm{z} 1<k+1, L>, t, k, C, S, E\left\{N_{C}\right\} k_{S}$, $E\left\{h\left(\mathrm{z} 1^{\prime}<1, k>, \mathrm{z} 1<k+1, L>, t, k, C, S, E\left\{N_{C}\right\} k_{S}\right)\right\} k_{C}^{-1}$}

Message 3 from the client puzzle protocol should be enhanced before it can be used in our authentication protocol. In message 3 of the client puzzle protocol, $\left\{\mathrm{z} 1^{\prime}<1, k>, \mathrm{z} 1<k+1, L>, t, k, C\right\}$ is the message that needs to be passed to the server $S$. To authenticate the client to the server, the client produces a signature of the message using its private key, i.e. $E\left\{h\left(z 1^{\prime}<1, k>\right.\right.$, $\mathrm{z} 1<k+1, L>, t, k, C, S)\} k_{C}^{-1}$. We emphasize here that the plaintext counterpart (i.e. $\left.\left\{\mathrm{z} 1^{\prime}<1, k>, \mathrm{z} 1<k+1, L>, t, k, C, S\right\}\right)$ of this encryption must also be submitted to the server. Although the client can still authenticate to the server by sending only the encryption output $E\left\{\mathrm{z} 1^{\prime}<1, k>, \mathrm{z} 1<k+1, L>, t, k\right.$, $C, S\} k_{C}^{-1}$, the server will then need to perform a computationally expensive public-key decryption operation in order to retrieve the puzzle solution and the timestamp for verification. Denial-of-service attack can then be achieved by repetitively sending bogus encryption outputs to trigger the server executing the decryption operations repetitively. So the plaintext counterpart should also be submitted to allow the server to perform the necessary verifications, like the client puzzle protocol, before performing the expensive public-key decryption in order to authenticate the client.

To prevent replay attack, a nonce $N_{C}$ is randomly generated and sent to the server. This nonce serves as a challenge to the server, which is to be returned in the next message. It is signed using the client's private key to form $E\left\{h\left(E\left\{N_{C}\right\} k_{S}\right)\right\} k_{C}^{-1}$. This signature can be combined with the previous signature to form $E\left\{h\left(\mathrm{z} 1^{\prime}<1, k>, \mathrm{z} 1<k+1, L>, t, k, C, S, E\left\{N_{C}\right\} k_{S}\right)\right\} k_{C}^{-1}$. This enhanced message 3 of the client puzzle protocol forms the message that should be sent in the third flow of the authentication protocol.

On the server side, the server performs as similar set of verifications like those in the client puzzle protocol as shown in Figure 1. If $k=0$, no solution needs to be verified and the verification $h\left(\mathrm{z} 1^{\prime}<1, k>, m<k+1, L>\right)=h(m)$ can be omitted. After that, the signature of the client is verified.

\section{Message 4: $S \rightarrow C: S, C, E\left\{N_{C}\right\} k_{S}, E\left\{h\left(S, C, E\left\{N_{C}\right\} k_{S}\right)\right\} k_{S}^{-1}$}


The remaining task of the protocol is to authenticate the server to the client. The client challenge $E\left\{N_{C}\right\} k_{S}$ is signed by server's private key in order to authenticate the server to the client.

On the client side, if the signature on message 4 is verified to be correct, the server is said to be authentic to the client. Figure 2 shows the overall picture of the proposed authentication protocol. If the submitted solution is incorrect, the execution of the protocol will be terminated.

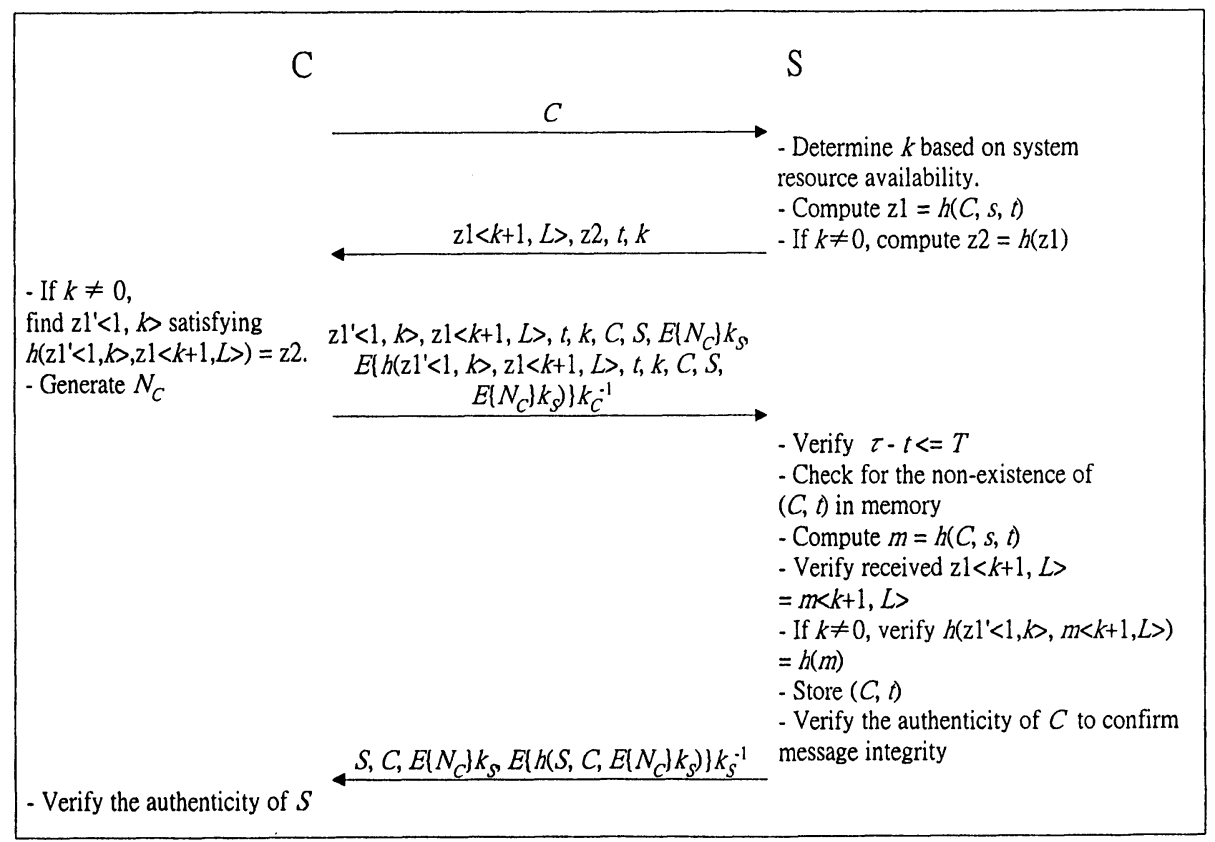

Figure 2. The DoS-resistant public-key authentication protocol

\subsection{Security Analysis}

The logic of the proposed authentication protocol has been analyzed and verified formally using the Coffey-Saidha technique [14]. In this section, the protocol is further analyzed against the common set of attacks on an authentication protocol [15].

\subsubsection{Denial-of-Service Attack}

This section analyzes the proposed authentication protocol to check if an unauthenticated client (or attacker) could exhaust server resources.

In our authentication protocol, the server receives two messages from the client. After the reception of message 1, no expensive computations are needed. The construction of the puzzle requires just two relatively fast hash 
operations. Also, the puzzle is constructed in a stateless fashion; so no memory resource is consumed. After the reception of message 3, computationally expensive public-key operations are needed to verify the client signature, and memory allocation is required to store the $(C, t)$ pair. However, these resources will only be consumed after the submitted solution has been verified correct, i.e. the client commitment of its resources has been assured. The non-existence checking of $(C, t)$ and the timestamp checking prevent an attacker from using a previously submitted solution. Therefore, the proposed authentication protocol ensures that the server will not be required to commit its extensive resources before confirming that the client has consumed some of its resources during the authentication protocol session. Further, an attacker could experience the risk of exhausting its own resources if it keeps on issuing authentication requests to the server. However, the server will assign only one copy of its memory resource to an authenticated client.

\subsubsection{Replay Attack}

Replay attack refers to those attacks involving the use of information from a single previous protocol execution, on the same or a different verifier [15].

The basic techniques to avoid replay attack include the use of the challenge-response technique; the use of nonces; and the embedding of target identity in a response message [15].

In a public-key cryptographic protocol, the potential messages that an attacker may replay are those that the attacker is unable to produce. In our authentication protocol, messages 3 and 4 are two such messages as they are encrypted using private keys unknown to the attacker.

In message 3 of the proposed authentication protocol, there is a bit-string $z 1<k+1, L>$ embedded inside the encryption using private-key $k_{C}{ }^{-1}$. This bitstring is generated and sent by the server to the client in message 2 of the protocol. It is unpredictable to the client as it is generated using one-way hash function with a long server secret key $s$ and current timestamp $t$ as inputs. Also, as shown in Section 2.2, the length of $z 1<k+1, L>$ must be sufficiently long (at least 64 bits) for the server to detect a fake timestamp. The bit-string $\mathrm{z} 1<k+1, L>$ thus serves the purpose of a nonce for the server to ensure the freshness of message 3 and so the replay attack is prevented.

The replay of message 4 is prevented by challenge-response technique. A long random nonce $N_{C}$ is generated and sent by the client to the server in message 3. It is then embedded inside the signature of message 4 in order to assure to the client the freshness of this message. 


\subsubsection{Interleaving Attack}

Interleaving attack refers to those attacks involving selective combination of information from one or more previous or simultaneously ongoing protocol executions (parallel sessions), including possible origination of one or more protocol executions by an adversary itself [15].

To compromise the proposed protocol using interleaving attack, the attacker must be able to derive those ciphertexts produced using the unknown private keys. In the proposed protocol, none of these ciphertexts in any of the protocol messages has the same format as the other three protocol messages or their components. Also, none of them has a format, which is a combination of the formats of the other three messages and their components. This makes the attacker unable to derive the desirable ciphertexts in one message from the other 3 messages of our protocol for an interleaving attack. If the attacker uses the $i^{\text {th }}$ protocol message of a previous protocol instance as the $i^{\text {th }}$ protocol message in the current instance, this corresponds to the replay attack, which has been addressed in Section 3.3.2 above. Another possible mode of interleaving attack is by forced delay [15], in which an attacker intercepted the $i^{\text {th }}$ protocol message of one protocol instance, relayed it at some later point in time and then uses it as the $i^{\text {th }}$ protocol message of another protocol instance in order to achieve impersonation or other deceptions. However this is not considered as a true attack because the attacker acts only as part of the communication link between the communicating parties without altering the content and aliveness of the protocol messages [15].

\subsubsection{Others}

As all the four protocol messages are asymmetric to each other, with identities of the sender and receiver embedded inside the cryptographic messages, together with the use of asymmetric keys, reflection attack [15] is thus prevented in the proposed protocol. Besides, the man-in-the-middle attack can also be easily prevented in the protocol as this attack can be foiled by the adoption of certificate-based public-key signature [16].

\section{CONCLUSIONS}

This paper proposes a client puzzle based authentication protocol which can effectively resist the denial-of-service attacks, and other common contemporary attacks. The integrated authentication protocol relies on our carefully designed client puzzle protocol, which can be seen as an enhanced 
version of the client puzzle proposed by Juels and Brainard [5], to tackle the network denial-of-service attack. Our client puzzle protocol has overcome problems inherent in the proposal of Juels and Brainard.

Concerning our proposed integrated authentication protocol, for each protocol execution request from a client, a unique puzzle would be generated and sent by the server to the client. The client must commit its resources to solve the puzzle before expensive computations and memory allocations would be performed by the server. This cost of attack serves as a deterrent to the attacker in carrying out server resource exhaustion attacks. The proposed client puzzle protocol is quite flexible as the difficulty level of the puzzle can be adjusted according to the degree of availability of the server resources. This means that if the attackers manage to consume some of the server resources, the server would increase accordingly the difficulty level of the client puzzles which would subsequently consume more resources of the attackers. Therefore, the protocol could intelligently monitor the attackers and launch counter through adjusting adaptively the puzzle difficulty level.

The proposed authentication protocol remains stateless and refuses to perform expensive public-key cryptographic computations until the solution of the client puzzle has been verified. Thus it can resist server resource exhaustion attacks. Besides, the proposed authentication protocol has been able to resist various common attacks. As the DoS attacks have become widely known and can easily be exploited to attack websites and various network protocols, they have created a real problem to protocol design. Protocols vulnerable to DoS attacks could be strengthened with the integration of our proposed client puzzle protocol. In addition, network protocol designers should consider integrating the proposed client puzzle protocol into their protocols to avoid being harassed by the DoS attackers.

\section{REFERENCES}

[1] CERT Coordination Center, "Denial of Service Attacks," Tech Tips, June 4, 2001. Available: http://www.cert.org/tech_tips/denial_of_service.html

[2] Garber, L., "Denial-of-service attacks rip the internet," IEEE Computer, vol. 33, Issue 4, pp. 12 -17, April 2000.

[3] Jussipekka Leiwo, Pekka Nikander, Tuomas Aura, "Towards network denial of service resistant protocols," in Proceedings of the $15^{\text {th }}$ International Information Security Conference (IFIP/SEC 2000), Beijing, China, August 2000. Kluwer.

[4] CERT Coordination Center, "TCP SYN Flooding and IP Spoofing Attacks," CERT Advisory CA-1996-21, September 19, 1996. Available: http://www.cert.org/advisories/CA-1996-21.html 
[5] Ari Juels and John Brainard, "Client puzzles: A cryptographic countermeasure against connection depletion attácks," in Proceedings of the 1999 Network and Distributed System Security Symposium (NDSS '99), pp. 151-165, San Diego, California, February 1999.

[6] Alan O. Freier, Philip Karlton and Paul C. Kocher, "The SSL Protocol Version 3.0," Internet Draft, March 1996

[7] Li Huang Ng and Tan. D.T., "A novel JavaCard-based authentication system for secured transactions on the Internet," in Proceedings of 2000 IEEE International Conference on Networks (ICON 2000), pp. 262-266, 2000.

[8] S. Hirose and K. Matsuura, "Enhancing the Resistance of a Provably Secure Key Agreement Protocol to a Denial-of-Service Attack," in Proceedings of the 2nd International Conference on Information and Communication Security (ICICS'99), pp.169-182, Sydney, Australia, November 1999. Springer.

[9] S. Hirose and K. Matsuura, "Enhancing the resistance of a secure key agreement protocol to a denial-of-service attack," in Proceedings of the 1999 Symposium on Cryptography and Information Security (SCIS'99), Vol. II, pp. 899-904, Jan. 1999.

[10] K. Matsuura and H. Imai, "Protection of Authenticated Key-Agreement Protocol against a Denial-of-Service Attack," in Proceedings of 1998 International Symposium on Information Theory and Its Applications (ISITA'98), pp. 466-470, Oct. 1998.

[11]Cynthia Dwork and Moni Naor, "Pricing via processing or combatting junk mail," in Ernest F. Brickell, editor, Advances in Cryptology -- CRYPTO '92, Volume 740 of Lecture Notes in Computer Science, pp. 139-147, 16-20 August 1992. Springer-Verlag, 1993.

[12] Tuomas Aura, Pekka Nikander, and Jussipekka Leiwo, "DOS-resistant authentication with client puzzles," in Bruce Christianson, Bruno Crispo, and Mike Roe, editors, Proceedings of the 8th International Workshop on Security Protocols, Lecture Notes in Computer Science series, Cambridge, UK, April 2000. Springer-Verlag.

[13]Hans Dobbertin, "Cryptanalysis of MD4," Fast Software Encryption, Third International Workshop, Volume 1039 of Lecture Notes in Computer Science (D. Gollmann, ed.), pp. 53-69. Springer-Verlag, 1996.

[14]T.Coffey, and P.Saidha, "Logic for verifying public-key cryptographic protocols," IEE Proceedings in Computers and Digital Techniques, vo1. 144, Issue 1, pp. 28-32, January 1997.

[15] Alfred J. Menezes, Paul C. van Oorschot and Scott A. Vanstone, Handbook of Applied Cryptography, CRC Press, 1997.

[16]Dong Hwi Seo and P. Sweeney, "Simple authenticated key agreement algorithm," IEE Electronics Letters, vol. 35 No. 13, June 1999. 